\title{
Proof Pearl: Proving a Simple Von Neumann Machine Turing Complete
}

\author{
J Strother Moore \\ Dept. of Computer Science, University of Texas, Austin, TX, USA \\ moore@cs.utexas.edu \\ http://www.cs.utexas.edu
}

\begin{abstract}
In this paper we sketch an ACL2-checked proof that a simple but unbounded Von Neumann machine model is Turing Complete, i.e., can do anything a Turing machine can do. The project formally revisits the roots of computer science. It requires re-familiarizing oneself with the definitive model of computation from the 1930s, dealing with a simple "modern" machine model, thinking carefully about the formal statement of an important theorem and the specification of both total and partial programs, writing a verifying compiler, including implementing an X86like call/return protocol and implementing computed jumps, codifying a code proof strategy, and a little "creative" reasoning about the nontermination of two machines.
\end{abstract}

Keywords: ACL2, Turing machine, Java Virtual Machine (JVM), verifying compiler

\section{Prelude}

I have often taught an undergraduate course at the University of Texas at Austin entitled A Formal Model of the Java Virtual Machine. In the course, students are taught how to model sophisticated computing engines and, to a lesser extent, how to prove theorems about such engines and their programs with the ACL2 theorem prover [5]. The course starts with a pedagogical ("toy") JVM-like model which the students elaborate over the semester towards a more realistic model, which is then compared to an accurate JVM model[9]. The pedagogical model is called M1: a stack based machine providing a fixed number of registers (JVM's "locals"), an unbounded operand stack, and an execute-only program providing the following bytecode instructions ILOAD, ISTORE, ICONST, IADD, ISUB, IMUL, IFEQ, GOTO, and HALT, with unbounded arithmetic.

This set of opcodes was chosen to allow students to easily implement and verify some simple M1 programs. On the last class day before Spring Break, 2012, the students complained that it was very hard to program M1; that in fact, it was "probably impossible" to do "real computations" with it because it lacks a "less than" comparator and procedures 1$]$

${ }^{1}$ Such judgements are obviously naive and ill-informed; any machine with a branchif- 0 , a little arithmetic, and some accessible infinite resource is Turing Complete. 
My response was "Well, M1 can do anything a Turing machine do." But on my way home that evening, I felt guilty:

M1 is a pedagogical device, designed to introduce formal modeling to the students and inculcate the idea that expectations on hardware and software can often be formalized and proved. I shouldn't just say it's Turing Complete. I should show them how we can prove it with the tools they're using.

Fortunately, I had Spring Break ahead of me and thus was born this project.

\section{Source Files}

The complete set of scripts for this project are part of ACL2's Community Books. See the Community Books link on the ACL2 home page [6]. After downloading and installing the books visit models/jvm/m1/. See the README file there. References to *. lisp files below are for that directory. If you have a running ACL2 session you could (include-book "models/jvm/m1/find-k!" :dir :system) and (in-package "M1") to inspect everything with ACL2 history commands. This paper is a guide.

\section{Related Work}

Turing Completeness proofs for various computational models have been a staple of computer science since the time of Turing and Church. Mechanically checked proofs of other important theorems in meta-mathematics (the Church-Rosser theorem, the Cook-Levin theorem, and Gödel's First Incompleteness Theorem) are less common but have been done with several provers. Here I focus on mechanically checked formal proofs of the computational completeness of a programming language.

As far as I am aware, the first and only such proof was done in 1984 [2, when Boyer and I proved that Pure Lisp was Turing Complete, using the prover that would become Nqthm. We were asked to prove completeness by a reviewer of [3], in which we proved that the halting problem for Pure Lisp was undecidable; the reviewer objected that we had not proved Pure Lisp Turing Complete.

An important distinction between 2] and the present work is that the "suspect" computational model in the former is the lambda calculus with general recursion (Pure Lisp), whereas here it is a very simple Von Neumann machine (or imperative programming language) similar to the contemporary JVM and its bytecode language 8 .

While I'm unaware of other mechanically checked proofs that a given programming language is Turing Complete, this work also involves proofs of properties of low-level assembly code and a verifying compiler. This tradition goes back at least as far as the mechanically checked proof of a compiler by Milner and Weyhrauch in 1972 [10. Highlights of subsequent systems verification work 
involving such mechanically checked reasoning include the "CLI verified (hardware/software) stack" of [1, and of course the even more realistic results of the seL4 microkernel [7] and VCC projects [4. But even with a verified program one must prove that the specification is Turing Complete.

\section{Turing Machines}

The present work uses the same Turing machine model as 2] (ported from Nqthm to ACL2) which was accepted by the reviewers of that paper. The model is based on Rogers' classic [12 formalization. A Turing machine description, tm, (sometimes called an "action table") is a finite list of 4-tuples or cells, $\left\langle s t_{\text {in }}\right.$, sym $\left., o p, s t_{\text {out }}\right\rangle$. Rogers represents a tape as a pair of half tapes, each being a (finite but extensible) list of $0 \mathrm{~s}$ and $1 \mathrm{~s}$. The concatenation of these two half tapes corresponds to the intuitive notion of a tape (extensible in both directions) with a read/write head "in the middle." Rogers shows one may start with an extensible finite tape. The read/write head is thought of as positioned on the first symbol on the right half. The interpretation of each cell in description $t m$ is "if, while in state $s t_{i n}$, sym is read from the tape, perform operation op on the tape and enter state $s t_{\text {out }}$." Here, $s t_{\text {in }}$ and $s t_{\text {out }}$ are symbolic state names, sym is 0 or 1 , and $o p$ is one of four values meaning write a 0 , write a 1 , shift left, or shift right. The machine halts when the current state and symbol read from the tape do not match any $s t_{i n}$ and $s y m$ in $t m$.

We define tmi ("Turing machine interpreter") to take a Turing machine state name, tape, and a Turing machine description and a number of steps, $n$. Tmi returns either nil ("false") meaning the machine did not reach a halted state in $n$ steps, or the final tape produced after $n$ steps. By our choice of tape representation, a tape can never be nil and so the function tmi indicates whether the computation halted in $n$ steps and the final tape if it did halt. See the definition tmi in tmi-reductions.lisp. I will colloquially refer to tmi as our "official" model of Turing machines.

In our official model, Turing machine descriptions and cells are lists constructed with cons, state names are Lisp symbols (e.g., Q1, LP, TEST), "symbols" on the tape are integers 0 or 1 , and operations are Lisp objects 0,1 , L, or R. See the definition of *rogers-program* in tmi-reductions.lisp for an example.

\section{$5 \quad$ M1}

M1 is defined in a similar style but takes an M1 state and a number of steps. An M1 state contains a program counter ("pc"), a list of integers denoting register values, a stack of integers, and a program; all components of an M1 state are represented with lists, symbols and numbers in the obvious way. The integers are unbounded, the stack may grow without bound. An arbitrary number of registers may be provided but the number of allocated registers never grows larger than the largest register index used in the program. Programs are finite and fixed ("execute only"). 
Programs are lists of the instructions as described below. The notation "reg[i]" denotes the contents of register (JVM local variable) $i$. "reg $[i] \leftarrow v$ " denotes assignment to a register; " $p c \leftarrow v$ " denotes assignment to the program counter. The notation " $\ldots, x, y, a \Rightarrow \ldots, v$ " describes the manipulation of the stack as per [8] and means that three objects, $x, y$, and $a$, are popped from the stack (with $a$ being the topmost) and $v$ is pushed in their place. That portion of the stack ("...") deeper than $x$ is unaffected. The first six instructions below always increment the pc by 1, i.e., $p c \leftarrow p c+1$ is implicit.

\begin{tabular}{|c|c|c|c|c|}
\hline instruction & & stack & & description \\
\hline (ILOAD $n$ ) & $\ldots$ & $\Rightarrow$ & $\ldots, \operatorname{reg}[n]$ & \\
\hline (ISTORE $n$ ) & $\ldots, v$ & $\Rightarrow$ & $\ldots$ & $\operatorname{reg}[n] \leftarrow v$ \\
\hline (ICONST $k$ ) & $\ldots$ & $\Rightarrow$ & $\ldots, k$ & \\
\hline (IADD) & $: \ldots, x, y$ & $\Rightarrow$ & $\ldots, x+y$ & \\
\hline (ISUB) & $: \ldots, x, y$ & $\Rightarrow$ & $\ldots, x-y$ & \\
\hline (IMUL) & $: \ldots, x, y$ & $\Rightarrow$ & $\ldots, x \times y$ & \\
\hline$($ GOTO $d)$ & $: \quad \ldots$ & $\Rightarrow$ & $\ldots$ & $p c \leftarrow p c+d$ \\
\hline$(\operatorname{IFEQ} d)$ & $: \quad \ldots, v$ & $\Rightarrow$ & $\ldots$ & $p c \leftarrow p c+($ if $v=0$ then $d$ else 1$)$ \\
\hline （HALT） & $: \quad \ldots$ & $\Rightarrow$ & $\ldots$ & no change to state \\
\hline
\end{tabular}

Note that by not changing the state, the HALT instruction causes the machine to stop. We consider an M1 state halted if the pc points to a HALT instruction.

To step an M1 state the instruction at pc in the program is fetched and executed as described above. We define (M1 $s n$ ) to step state $s n$ times and return the final state. See m1.lisp for complete details of the M1 model.

An example of an M1 program to compute the factorial of register 0 and leave the result on top of the stack is:

\begin{tabular}{|c|c|c|}
\hline program & $p c$ & pseudo-code \\
\hline '((ICONST 1$)$ & $; 0$ & \\
\hline (ISTORE 1) & ; & $\operatorname{reg}[1] \leftarrow 1$ \\
\hline (ILOAD 0) & ; & \\
\hline （IFEQ 10） & ; & if $\operatorname{reg}[0]=0$, then jump to 13 \\
\hline (ILOAD 1) & ; & \\
\hline (ILOAD 0) & ; & \\
\hline (IMUL) & ; & \\
\hline (ISTORE 1) & ; & $\operatorname{reg}[1] \leftarrow \operatorname{reg}[1] \times \operatorname{reg}[0]$ \\
\hline (ILOAD 0) & ; & \\
\hline (ICONST 1) & ; 9 & \\
\hline (ISUB) & ; 10 & \\
\hline (ISTORE 0) & ; 11 & $\operatorname{reg}[0] \leftarrow \operatorname{reg}[0]-1$ \\
\hline (GOTO -10) & ; 12 & jump to 2 \\
\hline (ILOAD 1) & ; 13 & \\
\hline$($ HALT $))$ & ; 14 & halt with reg[1] on top of stack \\
\hline
\end{tabular}

This program runs forever (never reaches the HALT) if $r e g[0]$ is negative.

If we require as a precondition that $r e g[0]$ is a natural number, a statement of total correctness can be paraphrased as: If $s$ is an M1 state with pc 0 , the natural number $n$ in $r e g[0]$ and the list above as the program, then there exists 
a natural number $i$ such that (M1 $s i$ ) is a halted state with $n$ ! on top of the stack.

To state and prove such a theorem it is convenient to define a witness for the existentially quantified $i$. This witness is delivered by a user-defined clock function that takes $n$ as an argument and returns a natural number.

The ACL2 Community Books directory models/jvm/m1/ contains many example M1 programs along with machine checked proofs of their correctness via such clock functions and other methods.

\section{The Correspondence Conventions}

To state Turing equivalence I followed the approach of 2]. Paraphrasing it into the M1 setting, I set up a correspondence between official Turing machine representations of certain objects (e.g., machine descriptions and state names) and their M1 representations. The former are composed of lists, symbols, and integers; the latter are strictly numeric.

Consider an arbitrary cell, $\left\langle s t_{i n}, s y m, o p, s t_{o u t}\right\rangle$, in a Turing machine description $\mathrm{tm}$. Given that $\mathrm{tm}$ contains only a finite number of state name symbols, we can allocate a unique natural to each and represent these naturals in binary in a field of width $w$ (which depends on the number of state names in $\mathrm{tm}$ ). We could represent each of the four possible op as naturals in 2 bits but we allocate 3 bits. Let the numeric encodings of the four elements of cell be $s t_{i n}^{\prime}, s y m^{\prime}, o p^{\prime}, s t_{\text {out }}^{\prime}$, respectively. Then the encoded cell is cell ${ }^{\prime}=s t_{i n}^{\prime}+2^{w} s y m^{\prime}+2^{w+1} o p^{\prime}+2^{w+4} s t_{\text {out }}^{\prime}$.

Using this convention we can represent a list of cells, $t m$, as follows. The empty list is represented as an encoded "cell" of 0s with $o p^{\prime}=4$ (using the otherwise unnecessary 3rd bit of $o p^{\prime}$ ). We call this value nnil. A non-empty list whose first cell is represented by cell' and whose remaining elements are recursively represented by tail is represented by cell ${ }^{\prime}+2^{4+2 w}$ tail.

The tape (which, recall, also encodes the read/write head "in the middle") is represented on M1 as two natural numbers, one specifying (via its binary expansion) the contents of the tape and the other specifying the head position

(via the number of bits in the left-half tape). Henceforth I use these conventions:

level variable value

Official tm : a Turing machine description

st : a Turing machine state name

tape : a Turing machine tape (with encoded head)

M1 $\quad w \quad$ : width of a state symbol encoding req'd by st and $t m$

nnil : the marked encoded "cell" $\left(o p^{\prime}=4\right)($ wrt $w)$

$\mathrm{tm}^{\prime} \quad$ : the M1 (numeric) representation of $t m$ (wrt $w$ and $n n i l$ )

$s t^{\prime} \quad:$ the M1 (numeric) representation of $s t$

tape' : the M1 (numeric) representation of tape contents

pos $^{\prime} \quad$ : the M1 (numeric) representation of the head position

$s_{0} \quad$ : the initial M1 state described below

\footnotetext{
${ }^{2}$ See [1].
} 
The initial M1 state $s_{0}$ is an M1 state with program counter 0 , thirteen registers set to 0 , the stack in which $s t^{\prime}, t a p e^{\prime}, p o s^{\prime}, t m^{\prime}, w$, and nnil have been pushed, and finally, as the program, a certain, fixed list of M1 instructions. That list of M1 instructions, called $\Psi$ and described below, is (allegedly) a Turing machine interpreter in the programming language of M1. Note that $s_{0}$ does not specify how long the Turing machine is to run.

The macro with-conventions in theorems-a-and-b.lisp formally defines these conventions. The macro binds the ACL2 variable s_0 (aka $s_{0}$ ) to the value above, in terms of the variables tm, st, and tape. Technically, it binds w, nnil, $\mathrm{tm}^{\prime}, \mathbf{s t}^{\prime}, \mathrm{tape}^{\prime}$, and pos $^{\prime}$ as specified in terms of $\mathrm{tm}, \mathrm{st}$, and tape, and binds s_o in terms of those auxiliary variables.

\section{Theorems Proved}

The discussion in [2] requires us to prove:

Theorem A. If tmi runs forever on st, tape, and $t m$ then M1 runs forever on $s_{0}$. More precisely, we phrase this in the contrapositive and say that if M1 halts on $s_{0}$ in $i$ steps then there exists a $j$ such that tmi halts in $j$ steps.

Theorem B. If tmi halts on st, tape, and $t m$ in $n$ steps, there exists a $k$ such that M1 halts on $s_{0}$ in $k$ steps and computes the corresponding tape.

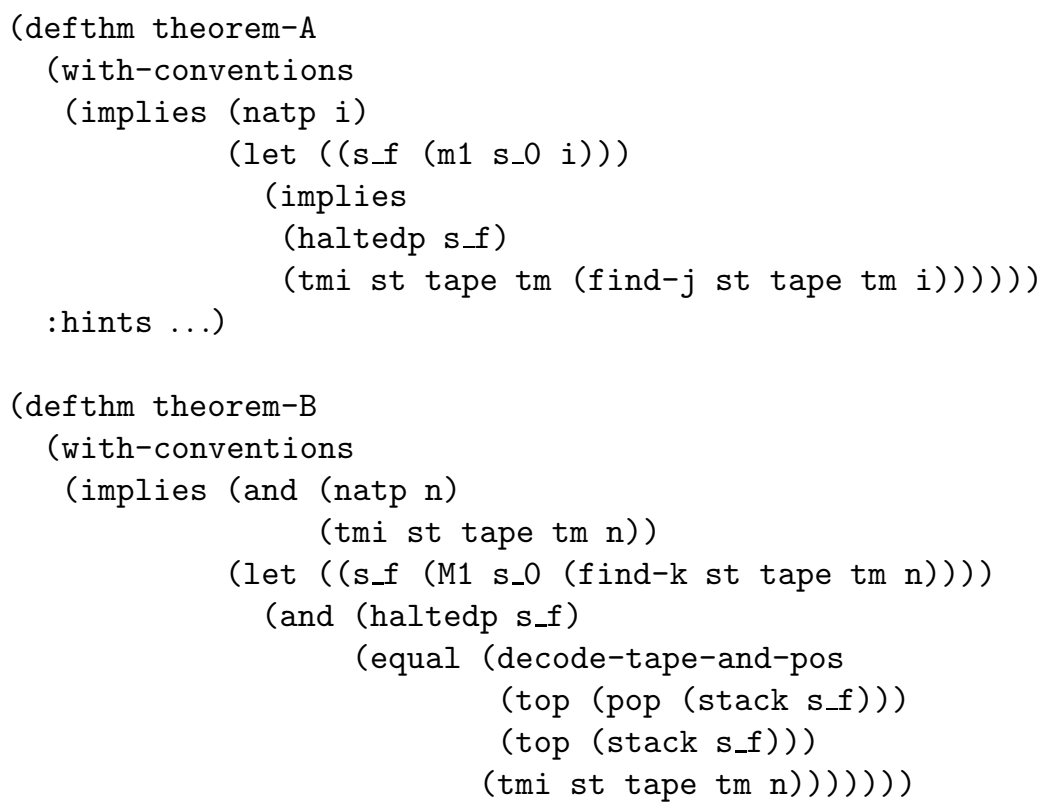

Note that when the tmi expressions are used as literals (e.g., in the conclusion of theorem-A and the hypothesis of theorem-B) it is equivalent to asserting termination (non-nil returned value) of the tmi run. When tmi is used in the equality, we know the value is a tape and the equality checks the correspondence with what M1 computes. 
In formalizing these statements there is an opportunity to subvert our goal by defining a devious sense of correspondence! The correspondence has access to the full power of the logic and could, for example, compute the right answer from tm, st, and tape and encode it into s_ 0 . The correspondence above is not "devious."

It remains to explain the fixed M1 program, $\Psi$, and the witness functions find $-j$ and $f$ ind $-k$ which constructively establish the existence of the step counts mentioned in the informal statements of the theorems. But first, it is convenient to refine tmi into a function that operates on the kind of data M1 has: numbers.

\section{Refinement}

We refine the official definition of tmi into a function named tmi3 and verify that it corresponds to tmi modulo the representational issues. The proof is done in several steps which successively implement the change of representations of tm and tape.

- tmi1 is like tmi but for a renamed $t m$ with numeric state names

- tmi2 is like tmi1 but for $t m^{\prime}, w$ and nnil

- tmi3 is like tmi2 but for tape' and pos'

This concludes with the theorem tmi3-is-tmi in tmi-reductions.lisp. It is tmi3 we will implement on M1.

\section{The M1 Program $\Psi$}

Key to our proof is the definition of an M1 program $\Psi$ for interpreting arbitrary Turing machine descriptions on a given starting state and tape. $\Psi$ either runs forever or HALTs; and when it halts, the representation of the official final tape can be recovered from the M1 state.

Given the limited instruction set of M1, it is necessary to implement some simple arithmetic utilities as M1 programs. $\Psi$ is then the concatenation of all these programs together with "glue code" permitting procedure call and return.

$$
\begin{aligned}
& \text { name stack description } \\
& \text { LESSP : } \quad \ldots, x, y \Rightarrow \ldots, v \quad v=(\text { if } x<y \text { then } 1 \text { else } 0) \\
& \underline{\mathrm{MOD}}: \quad \ldots, x, y \Rightarrow \ldots,(x \bmod y) \\
& \text { FLOOR : } \ldots, x, y, a \Rightarrow \ldots,(a+\lfloor x / y\rfloor) \\
& \underline{\mathrm{LOG} 2}: \ldots, x, a \Rightarrow \ldots,\left(a+\log _{2}(x)\right) \\
& \text { EXPT }: \ldots, x, y, a \Rightarrow \ldots,\left(a+x^{y}\right)
\end{aligned}
$$

We underline program names to help the reader; MOD names an M1 program, mod names an ACL2 function 3 . For brevity, the descriptions above do not include the effects of these programs on the pc or registers. In addition, certain obvious

\footnotetext{
${ }^{3}$ ACL2 is case insensitive; formally MOD is 'MOD
} 
preconditions obtain (e.g., for FLOOR, all operands are natural numbers and y is non-0).

With these primitives and subroutine call/return it is not difficult to define slightly higher level M1 programs for accessing encoded Turing machine descriptions, states, and tapes. The names below are all prefixed with ' $n$ ' because these functions are the numeric correspondents of functions in the official model of tmi. In the following, cell' is the numeric encoding of some cell $\left\langle s t_{i n}, s y m, o p, s t_{\text {out }}\right\rangle$, $s t_{i n}^{\prime}, s y m^{\prime}, o p^{\prime}, s t_{\text {out }}^{\prime}$ are the corresponding numeric encodings, $t m$ is assumed non-empty (and so its car is a cell with encoding $c a r^{\prime}$ and its $c d r$ is a list of cells with encoding $c d r^{\prime}$, and $t m^{\prime}$ is not nnil), $w$ is the width of the state symbol encoding, and nnil is the marked cell.

$$
\begin{aligned}
& \text { name : stack description } \\
& \underline{\text { NST-IN }}: \ldots, c e l l^{\prime}, w \Rightarrow \ldots, s t_{i n}^{\prime} \\
& \text { NSYM } \quad: \ldots, \text { cell }^{\prime}, w \Rightarrow \ldots, \text { sym }^{\prime} \\
& \text { NOP } \quad: \ldots, \text { cell }^{\prime}, w \Rightarrow \ldots, o p^{\prime} \\
& \text { NST-OUT }: \ldots, c e l l^{\prime}, w \Rightarrow \ldots, s t_{\text {out }}^{\prime} \\
& \underline{\mathrm{NCAR}}: \ldots, t m^{\prime}, w \Rightarrow \ldots, \text { car }^{\prime} \\
& \underline{\mathrm{NCDR}}: \ldots, t m^{\prime}, w \Rightarrow \ldots, c d r^{\prime}
\end{aligned}
$$

With these programs we can implement M1 programs for implementing the numeric version of tmi.

NCURRENT-SYM : ..., tape ${ }^{\prime}$, pos $^{\prime} \Rightarrow \ldots$, sym $^{\prime}$

Description: sym ${ }^{\prime}$ is the symbol at position pos $^{\prime}$ of tape'

NINSTR1 $: \ldots, a, b, \mathrm{tm}^{\prime}, w, n n i l \Rightarrow \ldots, c e l l^{\prime}$

Description: cell is the first encoded cell in $t m$ with $s t_{i n}^{\prime}=a$ and $s y m^{\prime}=b$, if any, or -1 if no such cell exists

NEW-TAPE2 $: \ldots, o p^{\prime}$, tape $^{\prime}$, pos $^{\prime} \Rightarrow \ldots$, tape $_{n x}^{\prime}$, pos $_{n x}^{\prime}$

Description: $o p^{\prime}$ is the encoding of a tape operation; tape $_{n x}^{\prime}$ and $p o s_{n x}^{\prime}$ are produced by performing that operation on tape ${ }^{\prime}$ and $p^{\prime} s^{\prime}$

$\underline{\text { TMI3 }}: \ldots, s^{\prime}$, tape $^{\prime}$, pos $^{\prime}, t^{\prime}, w$, nnil $\Rightarrow \ldots$, tape $_{n x}^{\prime}$, pos $_{n x}^{\prime}$

Description: This is the M1 program that interprets the Turing machine $t m$ with initial state st and input tape tape $e^{\prime}$ and pos $^{\prime}$. The program returns the tape $_{n x}^{\prime}$ and $\operatorname{pos}_{n x}^{\prime}$ representing the final tape if the machine halts, or runs forever otherwise.

Note that "tmi3" is both the name of an M1 program and of a function defined in ACL2 as part of our refinement of tmi to the M1 representations. However, the program TMI3 takes the six arguments listed above, while the function tmi3 takes an additional argument: the number of steps to take, n. The program TMI3 may run forever. The function tmi3 is total.

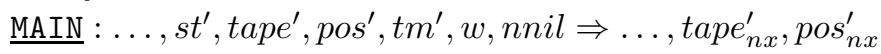

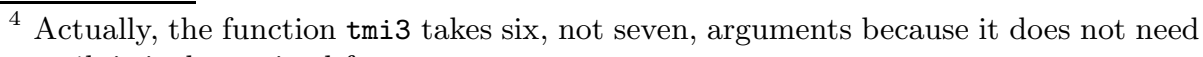
$n n i l$ : it is determined from $w$. 
Description: By convention, our compiler starts execution with the MAIN program and our MAIN just calls TMI3 above.

\section{Verifying Compiler}

Writing the sixteen programs above is tedious if done directly. Perhaps the main problem is that M1 does not support subroutine call and return: M1 operates on one "flat" program space! Furthermore, the machine does not provide "computed jumps" like the JVM's JSR (which pops the stack into the pc). There is a strict separation of data from pcs. Every GOTO and IFEQ is pc relative, but the distance skipped is always some constant specified in the instruction. Of course, writing the programs is only part of the battle: they must also be verified to implement the ACL2 function tmi3.

I thus decided to write a compiler from a simple "Toy Lisp" subset to M1 code. The compiler takes as input a system description, containing source code and specifications for every subroutine.

The verifying compiler is called defsys (see defsys.lisp). $\Psi$ is generated by the defsys expression in implementation.lisp. Every subroutine to be compiled is given a name, a list of : formals, an :input precondition, an : output specification describing the top of the stack, and the Toy Lisp source : code. It was sufficient and convenient to support only tail-recursive source code functions. As illustrated by main below, a subroutine may return multiple values and provision is made via so-called "ghosts" to model partial programs with total functions. Finally, optional arguments : $l d-f l g$ and : edit-commands allow the user to debug and modify the generated events. Inspection of implementation.lisp will reveal that three edit commands were used to augment the automatically generated commands. These generally inserted additional lemmas to prove before certain automatically generated theorems.

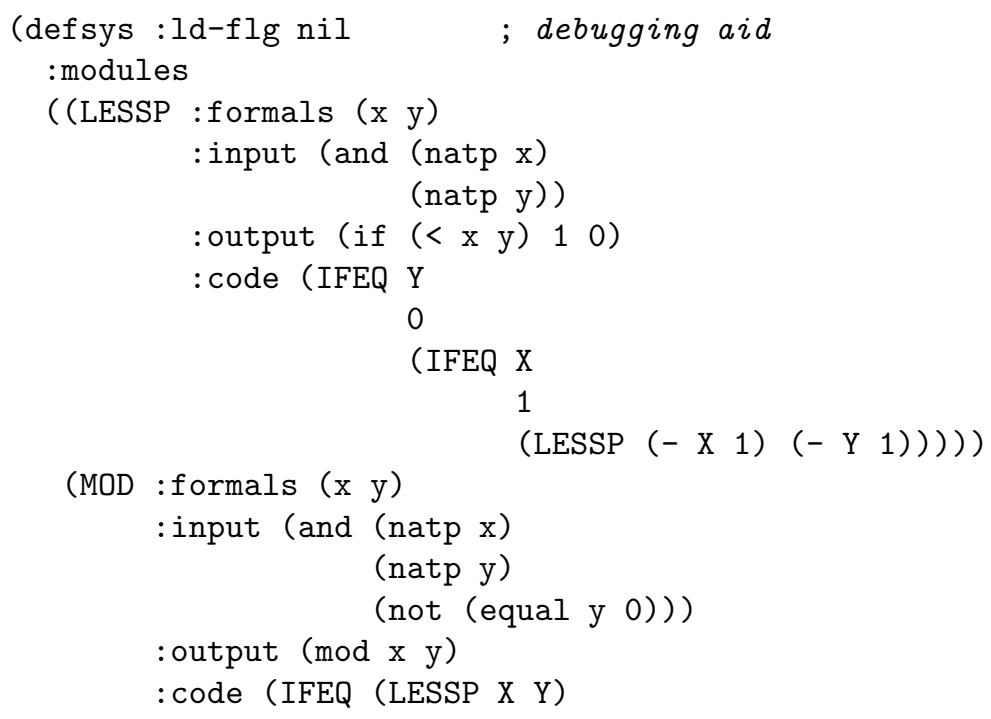




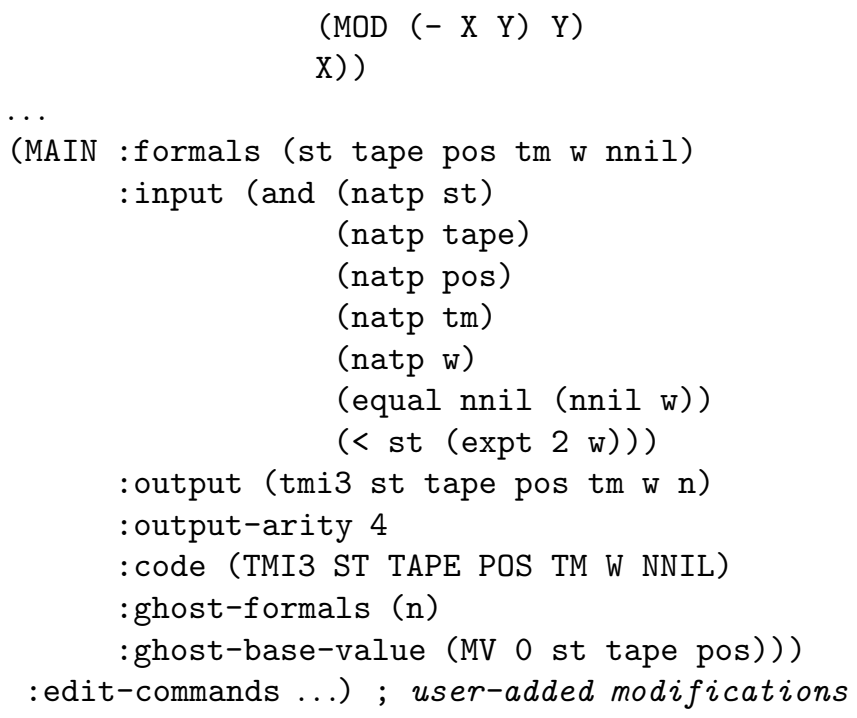

Toy Lisp is just the subset of ACL2 composed of variable symbols, quoted numeric constants, the function symbols,,$+- *$ (primitively supported by M1), the form (MV $a_{1} \ldots a_{n}$ ) for returning multiple values, the form (IFEQ $a b c$ ) (which is just ACL2's (if (equal $a r 0) \quad b \quad c$ )), and calls of primitive and defined Toy Lisp functions.

In addition to producing the M1 object code, the compiler (a) provides a call/return protocol, (b) links symbolic names to actual pcs (and generates appropriate relative jumps), and (c) produces the ACL2 commands (definitions and theorems) establishing that the object code is correct with respect to the Toy Lisp and that the Toy Lisp implements the :output specification.

If the maximum number of registers required by any subroutine's body is $\max$, the call/return protocol requires $2 \max +1$ registers. We divide them into $\max$ so-called A-registers, $\max +1$ B-registers. The A-registers are for use by the subroutine body and the B-registers are used by the call/return protocol. For simplicity we assume (and check) that the maximum number of registers used by a subroutine body is equal to the number of input parameters of the subroutine.

Note that of the sixteen programs sketched above, TMI3 has the most parameters: 6. Thus, we need 13 registers.

The basic protocol for calling a subroutine $\underline{s u b r}$ of $n$ arguments with arguments $a_{1}, \ldots, a_{n}$, is as follows: the caller pushes $a_{1}, \ldots, a_{n}$, and the $p c$ to which $\underline{s u b r}$ should return. The caller then jumps to the pc of $\underline{s u b r}$. At that pc, a prelude for $\underline{s u b r}$ pops $a_{1}, \ldots, a_{n}$, and $p c$ into the B-registers. It then protects the caller's environment by pushing the first $n$ A-registers onto the stack, followed by the return $p c$ from the B-registers. Finally, it moves the other B-registers (containing $\left.a_{1}, \ldots, a_{n}\right)$ to the first $n$ A-register 5 . A symmetric postlude supports returning

${ }^{5}$ The only way to move a value from one register to another is via the stack; only the topmost item on the stack can be accessed per instruction. 
$k \leq n$ values on the stack. At the conclusion of the postlude, the code jumps to the return $p c$.

But how can M1 jump to a pc found on the stack if the ISA firmly separates "data" from "pcs"? The answer is quite tedious: the compiler keeps track of every call of each subroutine; the postlude for each subroutine concludes with a "big switch" which compares the "return $p c$ " (data on the stack) to the known pc of each call and then jumps to the appropriate pc.

The compiler works in several passes. The first pass compiles the object code but includes symbolic labels and pseudo-instructions for CALL and RET. The second pass expands the CALL and RET "instructions" into appropriate sequences of M1 code. The last pass removes and replaces labels by relative jumps to the appropriate pcs. The compiler saves the output of the three passes in the ACL2 constants $*$ ccode $*$, *acode $*$, and $*$ Psi* respectively. These may be inspected after the compiler is run.

The key to generating the clock functions is just to count instructions in the prelude, loop, and postlude of each subroutine.

Defsys generates certain definitions and theorems for each subroutine, admits the definitions under the logic's definitional principle, and proves the theorems. The important ones are noted below for LESSH ${ }^{6}$. Recall that LESSP takes two arguments, $x$ and $y$. The : input condition of the module is that both $x$ and $y$ are naturals. The : output condition is that 1 or 0 is on top of the stack, depending on whether $x<y$. The source : code for the module is shown above. When $r p c$ is mentioned below it is the return pc from some call of LESSP in $\Psi$. When $s$ is mentioned it is an M1 state with program $\Psi$. Toy Lisp translations to ACL2 have names beginning with "!".

Def ( $! \operatorname{lessp} x y)$ : the ACL2 function !lessp is defined

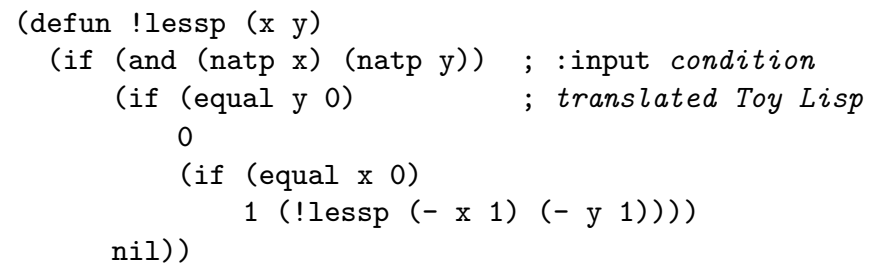

Def (lessp-loop-clock $x y$ ): defined to compute the number of M1 steps from the loop in LESSP to the postlude

Def (lessp-clock rpc $x \quad y$ ): defined to compute the number of M1 steps to get from the top of the prelude in LESSP through the return to rpc

Thm lessp-loop-is-! lessp: if the pc in $s$ is at the top of the loop in LESSP, with $x$ and $y$ (satisfying the stated : input conditions) in the first two A-registers, then after (lessp-loop-clock $x y$ ) steps the pc is at the postlude, all of the A-

\footnotetext{
${ }^{6}$ It is easiest to inspect the results by loading the project into ACL2 (Section 2) typing (pe 'name), where name is the name of an event mentioned here.
} 
registers except the first two are unchanged, and ( $\operatorname{lessp} x \quad y)$ has been pushed on the stack

Thm lessp-is-!lessp: if the pc in $s$ is poised at the pc of LESSP and the stack contains at least three values, $x, y$, and $r p c$, where $x$ and $y$ satisfy the : input conditions on lessp and $r p c$ is a known return pc from LESSP, then after (lessp-clock $r p c \quad x \quad y$ ) steps the pc is $r p c$, the A-registers are unchanged, and (! lessp $x y$ ) has been pushed onto the stack obtained by popping off $x, y$, and rpc

Thm !lessp-spec: if $x$ and $y$ satisfy the :input conditions for lessp, then (!lessp $x y)$ is as specified by the :output, i.e., it is 1 or 0 depending on $(x<y)$.

Putting the last two theorems together allows ACL2 to deduce that every jump to LESSP in $\Psi$ just advances the pc to the return pc, pops the arguments and the return pc off the stack, and pushes 1 or 0 according to the specification, without changing the A-registers.

Defsys compiles M1 code and generates and proves analogous definitions and theorems for every module. Thus, it compiles TMI3 and proves that running that code produces the results specified by tmi3. The only wrinkle in this story is that tmi3 takes a step-count argument while the program TMI3 does not. However, provision is made for this via the user-supplied "ghost" parameters of defsys. The clock function tmi3-clock and the : code function !tmi3 are augmented by an additional formal parameter, the user-supplied : ghost-formal $n$. In recursion (once per iteration), these functions decrement $n$ and halt if $n=0$. No such parameter exists in the compiled code. But defsys proves that the code, when run according to tmi3-clock, returns the same result as tmi3 (both wrt $n$ ), or else is left "still running" at the top of its loop.

\section{Finishing the Proof}

From the theorems in tmi-reductions.lisp we get that the official Turing machine interpreter, tmi, is equal to tmi3 modulo the representations, for any Turing step count $n$.

From implementation.lisp we get theorems about MAIN, its ACL2 analogue, !main and its : output specification function tmi3. In particular the theorem main-is-! main tells us that if invoked appropriately and run for main-clock M1-steps (for exactly $n$ iterations), the result is exactly described by its Lisp analogue !main: If !main reports halting after $n$ iterations, then the final M1 state has as its pc the return pc of the call of MAIN in $\Psi$, and the stack contains same tape and position computed by !main; and if !main reports that it did not halt (in $n$ iterations) the M1 state is poised at the top of the loop in the TMI3 program.

Meanwhile, !main-spec tells us that !main computes the same thing as tmi3.

Since main-clock starts counting from the pc of MAIN and $\Psi$ just pushes the return pc, jumps to $\underline{\text { MAIN }}$, and HALTs, we define (find-k st tape tm $n$ ) to be just 2 more than main-clock on the corresponding arguments $s t^{\prime}$, tape $^{\prime}$, pos $^{\prime}$, $t m^{\prime}, w$, nnil and $n$. 
In theorems-a-and-b.lisp we combine these results in the simulation theorem, which states that an M1 run starting in initial state $s_{0}$ and taking (find-k st tape $t m n$ ) steps is halted precisely if tmi halts in $n$ steps, and furthermore, that if tmi halts in $n$ steps, then the answer in the final M1 state corresponds to the tape computed by tmi.

Now we wish to prove theorem A and B. In fact, theorem-B (see page 6) follows easily from the simulation theorem.

Theorem A (page 6) requires more work. Recall that it deals with the nontermination of the two machines. Informally, it says that if tmi fails to terminate, then so does M1. But we phrased it in the contrapositive: if M1 terminates, then so does tmi.

Here we know that M1 halts on $s_{0}$ after $i$ steps and we must define $f$ ind $-j$ to return a number of steps sufficient to insure that tmi halts. Notice that the previously defined $\mathrm{f}$ ind-k counts M1-steps and now we seek to count tmi steps.

Two observations are important in defining $f i n d-j$. The first is a theorem called find-k-monotonic in theorems-a-and-b.lisp which states that if tmi has not halted after $n$ steps then (find-k st tape $t m n$ ) $<$ (find-k st tape $t m n+1)$. This is actually an interesting non-trivial theorem to prove, whose proof involved the only use of traces in the script.

The second observation is an easy one called m1-stays-halted: once M1 has halted, it stays halted. Thus, if M1 is halted after $i$ steps it is halted after any greater number of steps.

We can then define $\mathrm{find}-\mathrm{j}$ to find a $\mathrm{j}$ at which (tmi st tape $t m j$ ) is halted given that we know (M1 $s_{0} i$ ) is halted. The definition searches upwards from $j=0$ : if tmi is halted at $j$, return $j$; if (find-k st tape $\operatorname{tm} j$ ) $\geq i$, return $j$; else search from $j+1$.

This is a well-defined function: the recursion terminates because the find-k expression is growing monotonically and will therefore eventually reach the fixed $i$, if the earlier exit is not taken first.

It is easy to see that if M1 is halted at $i$, then $t m i$ is halted at ( $f$ ind $-j s t$ tape $t m i$ ): either find-j returns a $j$ (in the first exit) known to be sufficient or else it returns a $j$ such that (find-k st tape $t m j$ ) $\geq i$. But our second observation above shows that M1 must thus be halted at (find-k st tape tm $j$ ). And if M1 is halted there, then tmi must be halted at $j$, by the simulation theorem.

That completes our proof sketch of theorem A.

\section{Efficiency Considerations}

M1 is an executable operational model which ACL2 can execute at about 500,000 M1 bytecodes/sec. We can therefore run $\Psi$ to simulate Turing machines. The clock function find-k tells us exactly how long we must run it to simulate a given tmi run of $n$ steps.

Consider Rogers' Turing machine description for doubling the number on the tape [12. Suppose the tape starts with Rogers' representation for 4 on the tape. 
Running tmi experimentally reveals it takes 78 Turing steps to reach termination and compute a tape representing 8 . We can use $\mathrm{find}-\mathrm{k}$ to determine how long it takes M1 to simulate this computation. And the answer is:

$103,979,643,405,139,456,340,754,264,791,057,682,257,947,240,629,585,359,596$ or slightly more than $10^{56}$ steps!

The primary reason our implementation is so inefficient is that tapes and Turing machine descriptions are represented as large (bit-packed) integers and must be unpacked on M1 with programs that use LESSP. But the only way to answer the question "is $x<y$ ?" for two naturals $x$ and $y$ on M1 is to subtract 1 from each until one or the other becomes 0 , because the only test M1 programs can perform is equality against 0 . Thus it takes exponential time to unpack7.

The efficiency of our M1 Turing machine interpreter would be much improved if M1 provided the JVM instruction IFLT (branch if negative) or IF_ICMPLT (branch if $x<y$ ). Further improvement could be made by having IDIV (floor), or bit-packing operations like ISHR (shift right), IAND (bit-wise and), etc., and perhaps arrays (with IALOAD and IASTORE), to represent the tape. Minor further improvements could be had by supporting JSR or even INVOKESTATIC or INVOKEVIRTUAL to make call/return simpler. All of these features are supported on our most complete JVM model, M6 9 .

Another obvious approach would have been to compile the Turing machine description $\mathrm{tm}$ into an M1 program. Had I done so, the proofs of theorems A and $\mathrm{B}$ would have required proving that the Turing machine compiler was correct for all possible Turing machine descriptions. By representing Turing machine descriptions as data to be interpreted, I could limit my compiler's task to proving that its output was correct on the 16 Toy Lisp modules discussed. Put succinctly, it is easier to write a verifying compiler than to verify a compiler.

\section{Project History}

I developed M1 in 1997 to teach my JVM modeling course, which I subsequently taught about ten times. While the ISA of M1 changed annually to make homeworks harder or easier, programming M1 and proving correctness of my programs became almost second nature to me.

The question of M1's computational power arose in class in March, 2012. I completed the first version of this proof March 10-18, 2012 after coding $\Psi$ by hand in $804 \mathrm{M} 1$ instructions and manually typing the specifications and lemmas. I was helped enormously by the 1984 paper 2 and my experience with M1.

After Spring Break, I gave two talks on the proof: one to the Austin ACL2 research group and one to my undergraduate JVM class. Neither talk went smoothly and I learned a lot about the difficulty of presenting the work. A few weeks later, in early April, 2012, I decided to implement the verifying compiler. The present version of the proof was polished by April 14, 2012.

\footnotetext{
${ }^{7}$ And ACL2 would take exponential time evaluating find-k except for the theorems in find-k! .lisp
} 


\section{Conclusion}

Aside from the satisfaction of formally revisiting the roots of computer science, this work allowed me to go back into class after Spring Break and say:

M1 can do anything a Turing machine can do. Here's a proof.

\section{References}

1. W. Bevier, W. A. Hunt, Jr., J. S. Moore, and W. Young. Special issue on system verification. Journal of Automated Reasoning, 5(4):409-530, 1989.

2. R. S. Boyer and J. S. Moore. A mechanical proof of the turing completeness of pure lisp. In W. W. Bledsoe and D. W. Loveland, editors, Contemporary Mathematics: Automated Theorem Proving: After 25 Years, volume 29, pages 133-168, Providence, Rhode Island, 1984. American Mathematical Society.

3. R. S. Boyer and J. S. Moore. A mechanical proof of the unsolvability of the halting problem. Journal of the Association for Computing Machinery, 31(3):441-458, 1984.

4. E. Cohen, M. Dahlweid, M. A. Hillebrand, D. Leinenbach, M. Moskal, T. Santen, W. Schulte, and S. Tobies. Vcc: A practical system for verifying concurrent c. In Theorem Proving in Higher Order Logics, 22nd International Conference, TPHOLs 2009. Springer, 2009.

5. M. Kaufmann, P. Manolios, and J. S. Moore. Computer-Aided Reasoning: An Approach. Kluwer Academic Press, Boston, MA., 2000.

6. M. Kaufmann and J. S. Moore. The ACL2 home page. In http://www.cs.utexas. edu/users/moore/acl2/. Dept. of Computer Sciences, University of Texas at Austin, 2014.

7. G. Klein, K. Elphinstone, G. Heiser, J. Andronick, D. Cock, P. Derrin, D. Elkaduwe, K. Engelhardt, R. Kolanski, M. Norrish, T. Sewell, H. Tuch, and S. Winwood. seL4: Formal verification of an os kernel. In ACM Symposium on Operating Systems Principles, pages 207-220, October 2009.

8. T. Lindholdm and F. Yellin. The Java Virtual Machine Specification, 2nd edition. Prentice Hall, 1999.

9. H. Liu. Formal Specification and Verification of a JVM and its Bytecode Verifier. $\mathrm{PhD}$ thesis, University of Texas at Austin, 2006.

10. R. Milner and R. Weyhrauch. Proving compiler correctness in a mechanized logic. In Machine Intelligence 7, pages 51-72. Edinburgh University Press, 1972.

11. S. Ray and J. S. Moore. Proof styles in operational semantics. In A. J. Hu and A. K. Martin, editors, Formal Methods in Computer-Aided Design (FMCAD-2004), volume 3312 of Lecture Notes in Computer Science, pages 67-81. Springer, 2004.

12. H. Rogers. A Theory of Recursive Functions and Effective Commputability. McGraw-Hill, 1967. 